\title{
Frequency and Complications of Button Battery Cell as Foreign Body in Esophagus
}

Muhamamd Amer Nadeem, ${ }^{1}$ Muhammad Asim Shafiq ${ }^{2}$

\begin{abstract}
Background: Foreign bodies are commonly impacted in esophagus and button battery cells are one of the foreign bodies which could be found.

Objective: To find out the frequency and complications of button battery cell intake, among the cases of esophageal foreign bodies.

Methodology: A cross-sectional study was conducted at Department of ENT and Head and Neck Surgery Quaid-eAzam Medical College Bahawal Pur and Department of Otorhinolaryngology of Nishtar Hospital, Multan, from December 2017 to November 2018. After getting anesthesia fitness, rigid endoscopy was performed to remove the foreign body in 250 cases. Nasogastric intubation was done kept in place for two weeks before starting oral sips. Antibiotics, steroids and $\mathrm{H} 2$ receptor antagonists were given in cases of battery cell. Age, type of foreign body, endoscopic findings, level of impaction and type of complication were documented. Data was put in SPSS 23 and analyzed.
\end{abstract}

Results: Out of 250 cases of foreign bodies in esophagus, there were $50(20 \%)$ cases of button battery intake, with age range from 8 months to 8 years. The delay between ingestion and presentation ranged from 3 hours to 28 days. Out of 50 cases of button battery in esophagus, 20 (40\%) developed some complications; One patients developed tracheoesophageal fistula, 5 developed strictures, 1 developed hydropneumothorax, 2 developed vocal cord paresis, 1 developed stricture as well as aspiration pneumonia and 10 developed minor complication while one patient expired.

Conclusion: This study showed that button battery in esophagus is quite common in children, which warrants the prompt identification and removal of impacted battery to avoid complications.

Key words: Button battery, Esophagus, Management, Complication

Article Citation: Nadeem MA, Shafiq MA. Frequency and Complications of Button Battery Cell as Foreign Body in Esophagus. JSZMC 2020;11(2):08-12

\section{Introduction}

Foreign body (FB) impaction in the esophagus is considered a common emergency. Esophageal foreign body can be categorized as either true foreign body including blunt or sharp objects or food bolus. ' Impacted foreign body can be removed by flexible as well as rigid endoscopy. While presenting to hospital emergency or clinic, the presenting complaints vary significantly between the adults and children. Adults tend to describe the events and acknowledge the potential for esophageal foreign bodies. Children can be much vague in describing the complaints and in many cases (7\%-35\%) present with no symptoms. ${ }^{1}$

Disk battery cell or button batteries are matching with shape of coin, and are being used increasingly in the electrical and household gadgets such as television remotes, wrist watches, toys, electronic gadgets and hearing aids. ${ }^{1,2}$ These are now commonly presenting as esophageal foreign bodies in children younger than 5 years of age, and most disc batteries as FB esophagus are from hearing aids, watches, electronic games, toys and calculators. ${ }^{4}$ Battery causes mucosal damages by many ways including direct corrosive effect, electric burns, by direct leakage of chemical substances from the disc battery and by causing pressure necrosis of the underlying tissue. ${ }^{5-7}$ Majority of these factors cause liquefactive necrosis of the esophagus and it rapidly progresses to esophageal perforation when battery cell is impacted in esophagus, leading to severe and potentially fatal complication. ${ }^{6-8}$

According to a literature report, only $10 \%$ of battery cell ingestion cases present due to some symptoms while the rest of the cases remain asymptomatic and pass the disc battery in their stool within 2-7 days. ${ }^{8}$ Size, shape and type of foreign body are the major factors for impaction and major complications. As

1 Department of ENT, Nishtar Medical University and Hospital, Multan, Pakistan.

2. Department of ENT, Quaid Azam Medical College and Bahawal Victoria Hospital, Bahawalpur, Pakistan.

Correspondence: Dr. Muhammad Amer Nadeem, Associate Professor, Department of ENT, Nishtar Medical University and Hospital, Multan, Pakistan. 
far as the battery cell is concerned, the status of battery whether old or used, residual power or voltage and time elapsed between impaction and removal are the main determinants of morbidity and mortality. ${ }^{9}$ Fatal outcome or complications of battery cell is perforation of esophagus at the site of impaction which can be life threatening requiring active management. Either surgical repair of perforation or conservative management with antibiotics and nasogastric intubation can be performed, increasing the duration of hospital stay as well as treatment cost. ${ }^{9,10}$ Earlier signs and symptoms of esophageal perforation are rise in temperature, increased heart rate, tachypnea and vomiting. Nonspecific signs viewed on plain chest radiograph are pleural effusion, pneumothorax and pneumomediastinum, and widening of mediastinum. Other minor complications with battery cell impaction are vocal cord paralysis, mediastinitis, and aspiration, pneumonia and lung abscess. Battery cells rarely need removal if passed beyond the esophagus; however if impacted in lower GI tract, can cause abdominal pain and tenderness, and daily inspection of stools or repeated radiographs of abdomen after one week are recommended to confirm passage of battery cell. ${ }^{10}$ Battery cell ingestion or impaction in the developed world is usually benign even with increasing incidence, whereas it creates a lot of trouble not only for the family but for the medical team, in the developing countries. The purpose of this study was to observe the frequency and rate of complications of disc battery impaction among the children, who present with esophageal foreign body impaction, at Nishtar Hospital Multan, a teaching and tertiary care hospital.

\section{Methodology}

This cross-sectional study was conducted in the Department of ENT and Head and Neck Surgery Quaid-e-Azam Medical College Bahawal Pur and Department of Otorhinolaryngology at Nishtar Medical University, Multan. Ethical approval was obtained from the Institutional Ethical Review Board of the hospital. This study was included 250 patients who presented with esophageal foreign body impaction. The duration of study was from December 2017 to November 2018. Informed consent was obtained from the adult guardians of all the patients. Complete clinical examination of all the patients was performed and it was made sure of that the airway was patent. PA view of X-ray chest and anteroposterior as well as lateral views of $\mathrm{X}$-ray soft tissue neck, were acquired. Of all the patients presenting with esophageal foreign body, only those who had ingested disc battery cell, were included for further data analysis in the study. Investigation which were needed for anesthesia fitness were also acquired. These included complete blood count, complete urine examination, random blood sugar, liver function tests and renal parameters. After getting anesthesia fitness, rigid endoscopy was performed to remove the disc battery. Esophagoscopic findings were documented. Endoscopic complications were dealt with accordingly. Nasogastric intubation was done under vision after suction clearance of necrotic slough and nasogastric tube was kept in place for two weeks before starting oral sips. Antibiotics, steroids and $\mathrm{H}_{2}$ receptor antagonists, were given in cases of battery cell and during this period, severity and types of complications were assessed and were managed accordingly. Age, type of foreign body, time delay since disc battery impaction to presentation, endoscopic findings, level of disc battery impaction and type of complication, were documented for each patient. All the data was put in SPSS version 23 and analyzed. Percentages were calculated for different age groups, type of foreign bodies, patients with impaction in upper or mid esophagus and various groups with time delay in presentation at hospital after foreign body ingestion.

\section{Results}

Out of total 250 cases, of esophageal foreign body impaction, there were $50(20 \%)$ cases of button battery intake. Out of these 50 cases of button battery, there were 20 males and 30 female. Minimum age was 8 months to a maximum of 8 years. Out of 50 cases, $10(20 \%)$ cases were up to 1 year of age, $5(10 \%)$ cases were from 1 to 1.5 years of age, $15(30 \%)$ cases were from 1.5 to 2 years of age, $15(30 \%)$ cases were of 2 to 4 years of age, $5(10 \%)$ cases were of 4 to 8 years of age. The battery cell was impacted in upper end of esophagus in $35(70 \%)$ cases and mid esophagus in $15(30 \%)$ cases. Delay in hospital presentation for seeking medical advice is important factor which increases the risk of 
complications. In our study, the delay between ingestion/impaction and presentation at hospital ranged from 3 hours to 28 days (Table-III).

Table I: Distribution according to delay in presentation

\begin{tabular}{|l|c|c|}
\hline \multicolumn{1}{|c|}{ Duration } & No. of cases & Percentage \\
\hline Within $1^{\text {st }} 12$ hours & 23 & $46 \%$ \\
\hline $13-24$ hours & 16 & $32 \%$ \\
\hline 1 day to $1^{\text {st }}$ week & 5 & $10 \%$ \\
\hline $2^{\text {nd }}$ week & 3 & $06 \%$ \\
\hline $3^{\text {rd }}$ week & 2 & $04 \%$ \\
\hline $4^{\text {th }}$ week & 1 & $2 \%$ \\
\hline
\end{tabular}

In $100 \%$ of cases, endoscopic findings were; necrosis with slough of burnt tissues in varying degrees depending on duration of impaction and type of battery. All patients had mucosal edema, mucosal discoloration, mucosal burns and necrotic tissue, which was gently debrided with suction. Esophageal perforation was not visualized in any case. Nasogastric tube was passed under vision in every case for upto 14 days. Postoperative treatment including; steroids, intravenous antibiotics, and proton pump inhibitors were started. Before starting oral feed, all patients were reassessed for any sign and symptoms of any complication and in suspected case barium swallow was done.

There were $30(60 \%)$ cases, who had ingested 20 $\mathrm{mm}$ button batteries and $20(40 \%)$ cases had ingested about $25 \mathrm{~mm}$ button batteries. The status of the batteries were old or used in $35(70 \%)$ cases while the batteries were new in $15(30 \%)$ cases. Complications directly attributable to endoscopic removal of foreign bodies are rare, and when complications occur it is difficult to determine whether the cause is endoscopy or foreign body itself. However as far as disc battery impaction is concerned, most of the complications are due to foreign object itself.

Major to minor complications occurred in 20 patients $(40 \%)$ whereas benign outcome uneventful recovery was seen in $30(60 \%)$ patient and were discharged after period of observation. Out of major complications; 1 (5\%) patient developed trachea-esophageal fistula, which was treated with nasogastric intubation and antibiotics and it healed within two weeks of conservative management. Out of 20 patients having complications, strictures were seen in $5(25 \%)$ cases, which were treated with dilatation of the stricture twice with one week gap between dilatations; 1 (5\%) patient developed hydro pneumothorax and he was shifted to pediatric surgery unit for chest intubation and died after 2 days; vocal cord paresis was seen in $2(10 \%)$ patient, which improved with nasogastric intubation; and 1 (5\%) patient developed stricture as well as aspiration pneumonia with in first week of removal of battery cell, for which antibiotics and nebulization was started after consultation with pediatrician and dilatation of stricture was done once the patient had improved and started oral sips. This patient had presented after 28 days of impaction. Remaining 10 patients developed minor complications like laceration, disarticulations of milk teeth and sometimes bleeding in the oral cavity or through nasogastric tube. Of all the 20 patients, who had developed complications, mortality rate was observed to be $1(5 \%)$ in our study.

\section{Discussion}

Recent development of technology has increased the use of button batteries in electronic devices and is increasingly used in day to day life. ${ }^{11,12}$ First reported case of button battery ingestion was in 1977, who swallowed a camera battery which impacted in upper esophagus. ${ }^{13}$ Battery cells account for less than $2 \%$ of foreign bodies ingested in children. ${ }^{14,15}$ Initial reports in literature show that disc battery ingestion was leading to serious complications, because of delay in presentation or delayed endoscopic removal, while recent studies show decline in the rate of complications in the developed world (USA), but the situation in south Punjab area of Pakistan is of serious concern due to lack of awareness or delay in endoscopic removal. Esophago-pleural fistulae, pneumothorax, tracheo-esophageal fistulae, vocal cord paralysis and stricture are very frequently observed with disc battery impaction in the esophagus. Increased use of battery cell has increased its ingestion as a foreign body esophagus so public awareness about the problems of battery cell ingestion and complication must be launched to decrease the risk of morbidity and morbidity.

Awareness about risks is necessary for early diagnosis and prompt treatment to minimize complications. Cases of button battery ingestion typically present with irritability, food refusal dysphagia, and excessive salivation. Sometimes 
symptoms mimic lower airway involvement due to cough, strider or breathing difficulty. Button Battery should be included in differential diagnosis, if above symptoms or signs are present, furthermore, X-ray of neck shows radio-opaque shadow with double rim at the periphery unlike coin which has single rim. Diagnosis should always be made, if properly exposed X-Ray is taken. Button battery has distinctive bilaminar structure appear as double ring or double density in anteroposterior view. Sometimes in X-Ray chest, disk batteries similar to coins are easily mistaken as coins with serious consequences.

For a battery to get impacted in esophagus, it is to be of $20 \mathrm{~mm}$ or more in size. Batteries of smaller size usually pass through gastrointestinal tract. In review reports from 2000-2009, 92\% of disk batteries, with fatal outcome or major consequences were, $20 \mathrm{~mm}$ lithium cells. Lithium battery cells generate more current so are associated with more adverse effects. ${ }^{15,16}$ Liquefactive necrosis leading to perforation occurs within 2-6 hours of ingestion or impaction. ${ }^{15,16}$ Impaction of disc battery in esophagus is noted mostly in children younger than 5 years, ${ }^{17}$ which is also seen true in our study. Severe esophageal injuries occur in very short period. Esophageal corrosive injury and burn can occur as early as 2.5 hours after ingestion. But the perforation has been reported in 5 hours after ingestion of battery cell. ${ }^{1}$

In United States the National Battery Ingestion Hotline was created in 1982 and it has helped in reducing the incidence of major adverse outcomes. ${ }^{19}$ Manufacturers should redesign button batteries as leak-proof. ${ }^{20}$ In the study by Iqbal K, incidence of battery cell ingestion was 3 out of 155 total cases, ${ }^{21}$ while in our study it was 50 out of 250 cases, which shows increasing incidence. So it is very important to remove the battery cell as early as possible to reduce the risk of complication.

\section{Conclusion}

This study concluded that there is high frequency of cases of button battery ingestion impacted in esophagus among children, which is why there is need to identify and remove the impacted battery as early as possible to avoid serious complications.

\section{Author Contribution}

MAN: Conception of work, design of work and revising. MAS: Design of work, Acquisition and analysis of data and drafting.

All authors critically revised and approve its final version.

\section{Conflict of interest: None \\ Resources of funding: Self}

\section{References}

1. William W. W. Mnagement of foreign bodies of upper gastrointestinal tract. Gastrointest Endosc 1995; 41: 39-51.

2. Stanley J Kimball, Albert H, Park, et al. A review of foreign body disk battery ingestion and a protocol for management. Arch Otolaryngol Head Neck Surg. 2010; 136(9): 866-71.

3. LitovitzEL, Schmitz BF. Ingestion of cylindricle and button batteries; an analysis of 2382 cases. Pediatrics 1992; 89: 747-57.

4. Samad L, Ali M, Ramzi H. Button battery ingestion: hazards of esophageal impaction. Journal of Pediatric Surgery 1999; 34(10): 1527-1531.

5. Bass D.H, Millar A.J.W. Mercury absorption following button battery ingestion",Journal of Pediatric Surgery 1992; 27(2): 1541-1542.

6. Litovitz EL, Schmitz BF. Ingestion of cylindrical and button battery. An analysis of 2382 patients. Pediatrics 1992; 89: 747-57.

7. Chan YL, Chang SS, Kao KL, Liao HC, Liaw SL, Chiu TF, et al. Button battery ingestion: an analysis of 25 cases. Chang Gung Med J Mar 2002; 25 (3): 169-71

8. Lin V. Y., Daniel S. J., Papsin BC. Button battery in the ear, nose and aerodigestive tract. International Journal of Pediatric Otorhinolaryngology 2004:68; (4): 473-479.

9. Hamilton J. M., Schraff S A, D. M. Notrica. Severe injuries from coin cell battery ingestion: 2 case reports. Journal of Pediatric Surgery 2009; 44(3): 644-47.

10. Soerdjbalie-Maikoe V, van Rijn R. R. A case of fatal coin battery ingestion in a 2 year - old child. Forensic Science International 2010; 198(1): e19-e22.

11. Higo R, Matsumoto Y, Ichimura K, Kaga K. Foreign bodies in aerodigestive tract in pediatric patients. Auris Nasus Larynx 2003: 34 (4); 397-01.

12. Premachandra DJ, McRae D. Severe tissue destruction in ear caused by alkaline button battery. Post. Graduate Medical Journal 1990; 66(771): 52-53.

13. Blatnik DS, Toohnil R. J., Lehman R. H. Fatal complication from an alkaline battery foreign in the esophagus. Ann of Otology and laryngology 1977: 86 (5); 611-615.

14. Sheikh A. A button battery in children. Pediatric Emergency Care 1993; 9(4): 224-229.

15. Stanley J. Kimbal, Albert H. Park, et al. A review of esophageal disk battery ingestion and protocol for management. Arch. Otolaryngol Head Neck Surg 2010; 136(9): 866-871.

16. Slamon NB, Hertzog JH, Penfil SH, Raphaely RC, Pizarrro $\mathrm{C}$, Derby CD. An unusual case of button battery- induced traumatic tracheoesophageal fistula. Pediatr Emerg Case 2008; 24 (5): 313-16. 
17. Hossam M T, Mohamed W B, Askar S. Button battery Foreign bodies in Children: Hazards, Management and Recommendations. Biomedical Research International 2013: 1-7.

18. Srivastava G. Foreign bodies in the oropharynx gastrointestinal tract, ear, nose. Clinical Pediatric Emergency Medicine 2010: 11(2); 81-94.

19. Watson WA, Litovitz TL, Klein-Schwartz W, et al. 2003 annual report of the American Association of Poison Control Centers Toxic Exposure Surveillance System. Am J Emerg Med. Sep 2004; 22(5): 335-404.
20.Litovitz T, Whitakar N, Clark L. Preventing battery ingestion : an analysis of 8648 cases; Pediatrecs 2010: 125 (6): 1178-1183

21. Iqbql K, Khan MI. Esophageal foreign bodies: experience with rigid esophagoscope at D. I. Khan. Gomal J Med Sci 2012; 10: 194-7. 